\title{
Seizure Detection based on Spatiotemporal Correlation and Frequency Regularity of Scalp EEG
}

\author{
Yaozhang Pan, Cuntai Guan, \\ Kai Keng Ang, Kok Soon Phua, \\ Huijuan Yang, Dong Huang \\ Institute for Infocomm Research, \\ Agency for Science, Technology and Research \\ (A*STAR), Singapore
}

\author{
Shih-Hui Lim \\ Department of Neurology, \\ Singapore General Hospital, \\ and National Neuroscience Institute
}

\begin{abstract}
In this paper, a robust seizure detection system using scalp EEG signal is presented. Two most important and obvious characteristics of seizure EEG, signal variance, and frequency synchronization are carefully chosen as seizure detection indexes. To extract the representation of EEG variance, a spatiotemporal correlation structure is constructed based on space-delay covariance matrices with multi-scale temporal delay. The frequency synchronization of EEG is represented by a regularity index derived from wavelet packet transform. The extracted representations are combined to form a high-dimensional feature vector with redundant information. In order to reduce the redundancy, feature selection is performed using mutual information (MI) based on best individual features. The optimized set of features form a more compact feature vector for each 2 -s epoch of multichannel EEG. Feature vectors are then classified into ictal or interictal class using a linear support vector machine (SVM). To evaluate the proposed seizure detection system, unbiased leaveone-session-out cross-validation using clinical routine EEG from 7 patients are performed in experiments. The proposed method obtains average accuracy of $91.44 \%$ and average latency of 6.82 s, which outperforms other 7 commonly used methods. It is also demonstrated that the performance of our method is more robust since the standard deviation of results among patients is smaller than other methods.
\end{abstract}

\section{INTRODUCTION}

Epilepsy is a common neurological disorder affecting approximately $1 \%$ of the world population. It is an electroclinical syndrome, characterized by paroxysmal electrical discharges of cortical neurons accompanied by clinical manifestation of epileptic seizures. Electroencephalography (EEG) is the diagnostic tool to detect these interictal epileptiform discharges and ictal electrographic seizures.

In the routine EEG laboratory, detection of electrographic seizures is done by EEG technologists and electroencephalographer who have to go through multi-cannel EEG data visually. This process is both time consuming and laborious. Seizures might be missed if the technologists and/or EEGer are inexperienced. Automatic detection of electrographic seizures by a computer software will make the detection of electrographic seizures much easier and more accurate. In the ICU setting, automatic detection of EEG seizures could help medical and nursing teams to administer immediate intervention to patients developing seizures under monitoring.
In this preliminary research, we investigate the fundamental mechanism of the seizure onset in order to develop a reliable seizure detection system. Based on the fact that during seizures scalp EEGs could record rhythmic synchronized paroxysmal EEG activities, we proposed the hypothesis that at the onset of seizure,

i) EEG variance across different channels is relatively small;

ii) EEG time-dependent variance in each channel is relatively small;

iii) EEG power is concentrated in a small range of lowerfrequency components compare to normal EEG.

We aim to find a reliable method for automatic seizure detection based on the characteristics mentioned above. Reliable and sensitive reflections of above characteristics of seizure EEG can be obtained using feature extraction algorithms. Several types of features have been investigated to discriminate EEG signal between ictal and interictal states. Some univariate features such as the power spectral density [1], entropy [2], median absolute deviation (MAD) [3], wavelet coefficients [4] and autoregressive modeling coefficients [5] for single-channel EEG were commonly used. In [6], [7], several univariate features, such as mean, curve length, energy, nonlinear energy and six power were combined together to form a hybrid higher-dimensional feature vector for identification of ictal and interictal state. However, due to the missing of correlation information across channels, the single-channel-based methods cannot provide satisfactory performance for seizure detection.

In [8], the authors utilized a multi-scale spatiotemporal correlation structure to extract variations of brain dynamic for predicting seizure using intracranial EEG. The multiscale spatiotemporal correlations were correlations among all channels and across temporal delay with multi-scale. It well represented the variance across different channels and different time epochs of each channel. Unfortunately, this method only considered the variance in time domain but ignored the important characteristics in frequency domain. From frequency domain point of view, EEG power is concentrated in a small range of relatively-low-frequency components during onset of seizures. In this work, to make good use of this characteristic, a frequency regularity index which represents the degree of 
EEG rhythmic synchronization is adopted. We combine the spatiotemporal correlation and the frequency regularity index to form a more robust seizure index, which is sensitive to both amplitude and frequency change. However, the combined seizure index is high-dimensional and contains redundant information. To reduce the redundancy, features selection are performed using mutual information (MI) based on best individual features with parzen window. The selected features form a more compact feature vector and classified into ictal or interictal class using linear SVM.

The main contributions of this work are:

i) By investigating the fundamental mechanism of epileptic seizure, we propose three most important characteristics of seizure EEG for guiding the development of seizure detection algorithm, and verify them through signal analysis.

ii) By combining the spatiotemporal correlation and frequency regularity index, a more robust seizure detection algorithm is developed, which is sensitive to both the frequency and amplitude(energy) variance of EEG signals.

iii) The detection results of 43 seizure data from 7 epilepsy patients demonstrate that our method obtains higher accuracy and robustness compare to the other 7 commonly used methods in literature.

\section{Methods}

\section{A. Overview}

The block diagram in Fig. 1 presents the architecture of the seizure detection procedure. The detector passes 2-second epochs of the 18-channel EEG through a bandpass filter of 0.5$30 \mathrm{~Hz}$. Feature extractor extracts spatiotemporal correlation and frequency regularity. A trained feature selector obtains features best characterizing the morphology of multi-channel EEG. Finally, the obtained features are passed into a trained SVM to get the identification result of current EEG epoch.

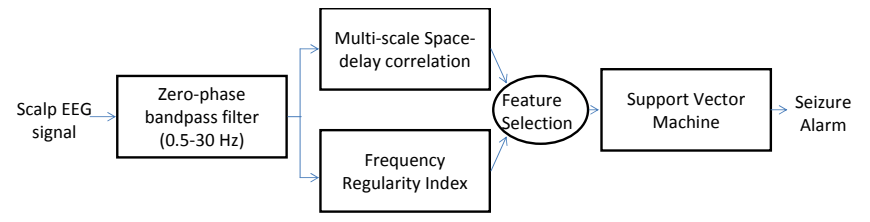

Fig. 1. Seizure Detector Architecture

\section{B. Data Recordings and Preprocessing}

To evaluate the performance of the proposed seizure detection algorithm, we use an EEG dataset provided by Singapore General Hospital (SGH) after ethics approval. The dataset contains routine EEG signals of 7 epilepsy patients, who are 3 females and 4 males aged between 28-77. The duration of each routine EEG recording session is around 30 to 50 minutes. The EEG signal was recorded using standard 10-20 system with sampling rate of $256 \mathrm{~Hz}$. Visually inspection of both EEG and video were undertaken by experienced technician for marking the seizure events. The labeling results can be served as ground truth in this work.
In Table I, the age, gender of the patients, and the duration and number of seizures are shown to demonstrate the characteristics of the EEG dataset.

TABLE I

EEG DATASET CHARACTERISTICS

\begin{tabular}{|c|c|c|c|c|}
\hline Subjects & Gender & Age & Duration(sec) & No. of Seizures \\
\hline 1 & Female & 38 & 1829 & 7 \\
2 & Female & 77 & 1839 & 2 \\
3 & Male & 70 & 1993 & 2 \\
4 & Female & 28 & 2225 & 3 \\
5 & Male & 54 & 1988 & 2 \\
6 & Male & 65 & 3872 & 22 \\
7 & Male & 47 & 3060 & 5 \\
\hline summary & N.A. & $58.22 \pm 17.45$ & $309.52 \mathrm{~min}$ & 43 \\
\hline
\end{tabular}

The EEG data are preprocessed via bandpass filtering between 0.5 and $30 \mathrm{~Hz}$, which is a frequency band that captures seizure onset of various electrographic manifestations [4], [9], [10]. The bandpass filter is designed to be a zero-phase to avoid phase shifting during filtering. Bipolar EEG montage which includes 18 channels is used to reduce the effect of common noise/artifact such as EMG etc. The continuous EEG recording is segmented into $2 \mathrm{~s}$ epochs with $1 \mathrm{~s}$ overlap by a moving window processing.

\section{Spatiotemporal Correlation with Multi-scales Delay}

Eigenspectrograms derived from spatial correlation matrices have been recently proposed for analyzing seizure dynamics in multichannel intracranial EEG signals [11]. Later, Willamson et. al extend it to multi-scales spatiotemporal correlation in [8] to do seizure detection in intracranial EEG signals. To the best of our knowledge, there has been no study yet applied this method to scalp EEG signals for seizure detection.

Furthermore, in [8], feature extraction was done using 15 $\mathrm{s}$ window of data, combine with the $15 \mathrm{~s}$ delay in the largest delay scale, the detection latency was at least $30 \mathrm{~s}$, which is not ideal for seizure detection since earlier detection and treatment of seizure is essential for preventing serious brain damage. In this work, we utilize a $2 \mathrm{~s}$ moving window plus 1 $\mathrm{s}$ delay in the largest delay scale, which dramatically shorten the detection latency. In addition, by carefully setting the delay scales, we eliminate the overlapping that come out from the setting in [8], which may reduce the feature redundancy.

The spatiotemporal correlation structure is constructed as following.

Consider $\boldsymbol{Y}(j)$ as the $j$ th epoch of the preprocessed multichannel EEG signals, $\boldsymbol{Y}(j) \in R^{n_{s} \times n_{c}}(512 \times 18$ in this study), where $n_{s}$ is the number of samples in each epoch and $n_{c}$ is the number of channels. Define $\boldsymbol{X}(j)$ as the second half part of each epoch $\boldsymbol{Y}(j), \boldsymbol{X}(j) \in R^{256 \times 18}$. As such, the start of each successive $1 \mathrm{~s}$ epoch $\boldsymbol{X}(j)$ is contiguous with the end of the previous epoch $\boldsymbol{X}(j-1)$. Define $\tau_{k}$ as the time delay in the $k$ th scale in unit of seconds. Then a set of time-delayed multichannel EEG signal can be written as

$$
\boldsymbol{Z}(j)_{k}=\left[\boldsymbol{X}(j), \boldsymbol{X}\left(j-\tau_{k}\right), \ldots, \boldsymbol{X}\left(j-\left(n_{d}-1\right) \tau_{k}\right)\right]
$$


where $\tau_{k}=\delta^{k}$ ( $\delta$ is the scale coefficient, here set as $1 / 4$ ), $k=0,1,2,3$. The number of time delay $n_{d}$ is set to be 2 for the largest scale and 4 for the other three scales, so that the delayed matrix will not exceed the $2 \mathrm{~s}$ duration of each epoch $\boldsymbol{Y}(j)$. By this way, we eliminate the overlapping that come out from the setting in [8].

These procedure result in a new multi-scale time-delayed matrix

$$
\boldsymbol{Z}(j)=\left[\boldsymbol{Z}(j)_{0}, \boldsymbol{Z}(j)_{1}, \boldsymbol{Z}(j)_{2}, \boldsymbol{Z}(j)_{3}\right]
$$

where

$$
\begin{aligned}
& \boldsymbol{Z}(j)_{0}=[\boldsymbol{X}(j), \boldsymbol{X}(j-1)] \\
& \boldsymbol{Z}(j)_{1}=\left[\boldsymbol{X}(j), \boldsymbol{X}\left(j-\frac{1}{4}\right), \boldsymbol{X}\left(j-\frac{1}{2}\right), \boldsymbol{X}\left(j-\frac{3}{4}\right)\right] \\
& \boldsymbol{Z}(j)_{2}=\left[\boldsymbol{X}(j), \boldsymbol{X}\left(j-\frac{1}{16}\right), \boldsymbol{X}\left(j-\frac{1}{8}\right), \boldsymbol{X}\left(j-\frac{3}{16}\right)\right] \\
& \boldsymbol{Z}(j)_{3}=\left[\boldsymbol{X}(j), \boldsymbol{X}\left(j-\frac{1}{64}\right), \boldsymbol{X}\left(j-\frac{1}{32}\right), \boldsymbol{X}\left(j-\frac{3}{64}\right)\right] .
\end{aligned}
$$

A space-delay covariance matrix $\boldsymbol{C}(j)_{k} \in R^{n_{c} n_{d} \times n_{c} n_{d}}$ and correlation matrix $\boldsymbol{R}(j)_{k} \in R^{n_{c} n_{d} \times n_{c} n_{d}}$ are calculated as

$$
\begin{aligned}
\boldsymbol{C}(j)_{k} & =\frac{1}{n_{s}} f\left(\boldsymbol{Z}(j)_{k}\right)^{T} f\left(\boldsymbol{Z}(j)_{k}\right), \\
\boldsymbol{R}(j)_{k} & =\frac{1}{n_{s}} g\left(\boldsymbol{Z}(j)_{k}\right)^{T} g\left(\boldsymbol{Z}(j)_{k}\right),
\end{aligned}
$$

where $f(\cdot)$ normalizes each column of $\boldsymbol{Z}(j)_{k}$ to zero mean, and $g(\cdot)$ normalizes each column of $\boldsymbol{Z}(j)_{k}$ to zero mean and unit variance.

Then we calculated the eigenvalues of $\boldsymbol{R}(j)_{k}$,

$$
\boldsymbol{\lambda}(j)_{k}=\operatorname{eig}\left(\boldsymbol{R}(j)_{k}\right),
$$

and order the eigenvalues from largest to smallest. These form the eigenspectrum of $\boldsymbol{R}(j)_{k}$.

Fig. 2 shows two epochs of 18 channel EEG signals of ictal and interictal state respectively. From the figure, we can clearly see the EEG signal difference between ictal and interictal state. Compare to interictal state, the EEG signal in ictal state has higher amplitude but smaller variance across time and channels, while the frequency components are more concentrated to a lower frequency range.

Fig. 3 shows the space-delay correlation matrix $\boldsymbol{R}(j)_{3}$ at the smallest delay scale, extracted from the two epochs shown in Fig. 2. Each $4 \times 4$ block along the main diagonal contains the within-channel correlations for the 4 time-delay scales for each of the 18 channels, while the off-diagonal blocks contain the cross-channel correlations. It is clearly illustrated in Fig. 3 that the cross-channel correlations are higher in interictal state than in ictal state, which indicate that the cross-channel variance is larger in interictal state. This is consistent with the i) and ii) of hypothesis we proposed in Section I.

Fig. 4 displays the eigenspectrogram with the smallest delay scales of a whole session of patient 1, which contains 1809 s EEG recording (we remove the first 20 seconds calibration data). Eigenspectrogram is a matrix consisting of a sequence

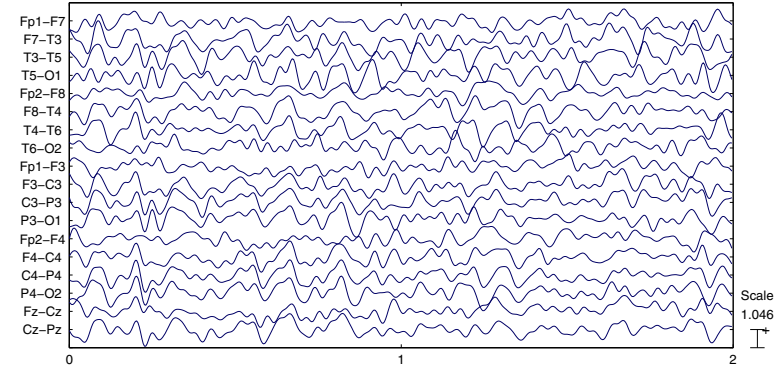

(a) Ictal state

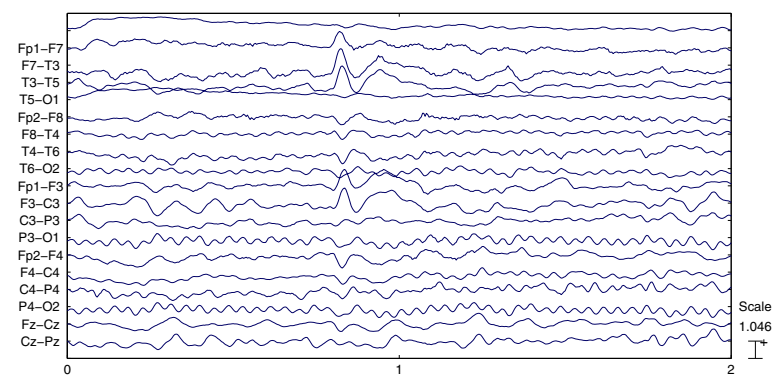

(b) Interictal state

Fig. 2. Two epochs of multichannel EEG: ictal and interictal

of eigenspectra. Each column of the matrix contains the eigenspectrum from a single epoch, and the bottom row contains the maximum eigenvalues.

Fig. 4 reveals much lower value of covariance across channels and time intervals during ictal states. This is consistent with the i) and ii) of hypothesis we proposed in Section I that variance across channels and time intervals is smaller in ictal state than in interictal state.

Eigenspectrums are also computed at the other 3 larger delay scales. For each epoch, 252 eigenvalues in total are derived from 4 delay scales and serve as the feature for representing the variance across channels and time intervals.

\section{Frequency Regularity Index}

Because EEG power is concentrated in a small range of relatively low-frequency components during seizure, the rhythmic regularity of the EEG signal can be considered as an indicator of ictal states. In this paper, we utilize a frequency regularity index of EEG signals motivated by [12], [13] to represent the rhythmic regularity of EEG signal.

To calculate the regularity index, it is necessary to find suitable frequency band that captures most significant rhythmic activities during ictal state. To make it simple, we choose the frequency range of 0.5 to $30 \mathrm{~Hz}$, as a large number of epileptic seizures are associated with rhythmic activities in this frequency range [9], [10]. Certainly, this frequency band 


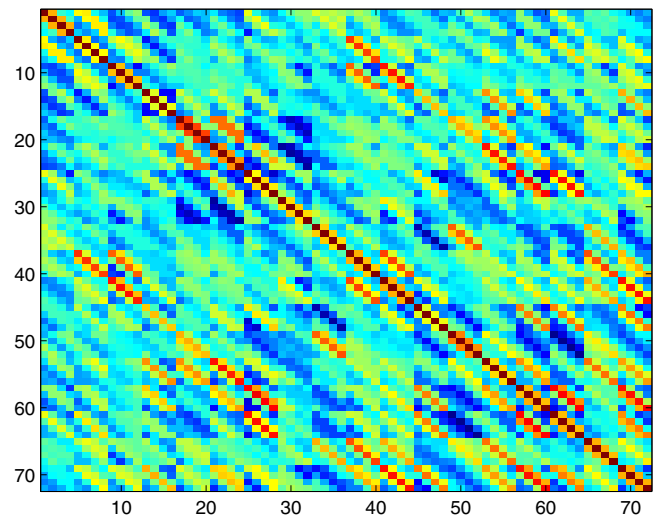

(a) Ictal epoch

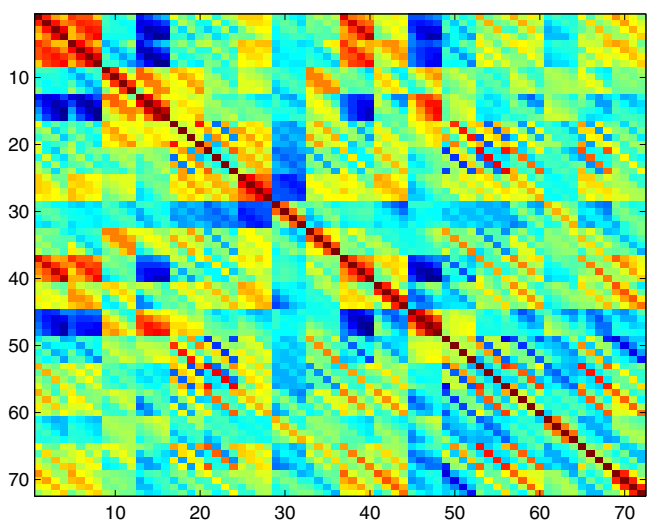

(b) Interictal epoch

Fig. 3. Space-delay correlation matrix

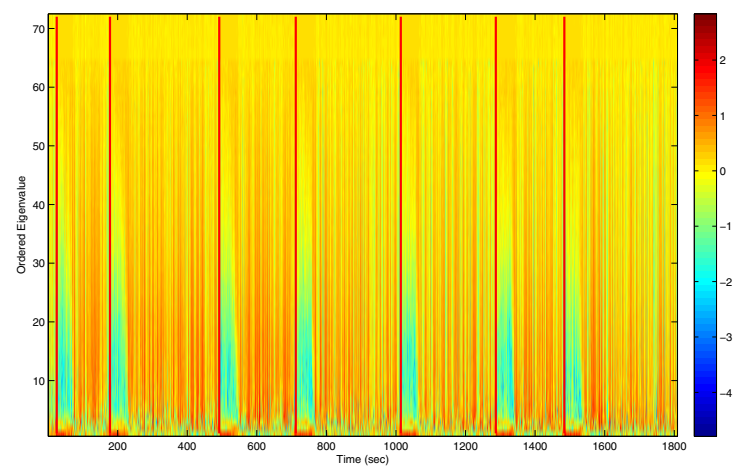

Fig. 4. Eignespectrogram of an entire session. Red markings indicate seizures manually marked by EEG technician. can be further defined subject-dependently to improve the performance.

The wavelet transform has well known data compression and time-frequency filtering capabilities and is promising for seizure detection [4]. Our method is based on the wavelet packet transform. Different from wavelet transform, the decomposition procedure of wavelet packet transform is done in both lower and higher frequencies, so that it offers a greater range of possibilities for signal analysis than the wavelet decomposition.

we use a moving window to obtain several $2 \mathrm{~s}$ EEG epoches from the original continuous EEG, and bandpass filter each epoch between 0.5 and $30 \mathrm{~Hz}$. Consider $\boldsymbol{Y}$ as the a $2 \mathrm{~s}$ epoch of the preprocessed multichannel EEG signals, $\boldsymbol{Y} \in R^{n_{s} \times n_{c}}$, where $n_{s}$ is the number of samples in each epoch and $n_{c}$ is the number of channels. Define $\boldsymbol{y} \in R^{n_{s} \times 1}$ as one channel EEG signal in the multichannel EEG epoch $\boldsymbol{Y}$. We decompose $\boldsymbol{y}$ using $l$ level wavelet packet (WP) transform within the frequency band $(0.5-30 \mathrm{~Hz})$.

The wavelet packet decomposition is done until the the last decomposition level with maximum frequency resolution. For a time epoch with sampling rate $F_{s}$ and window length $T$, the WP tree has $l=\log _{2} F_{s} * T$ decomposition level (excludes the root level). In our experiment, sampling rate $F_{s}=256$ $\mathrm{Hz}$ and window length $T=2 \mathrm{~s}$, so the last decomposition is 9th level. At the last level, there are 512 nodes each of which contains one coefficient associated with bandwith of $0.25 \mathrm{~Hz}$ $\left(F_{s} / 2^{l+1}\right)$.

Define $V_{l n}$ as the vector consists of all the coefficients at node $n, n=1,2, \ldots, 2^{l}$ in last decomposition level $l$ of the WP tree. From $\boldsymbol{V}_{l n}, n=1,2, \ldots, 2^{l}$, choose the node $\boldsymbol{V}_{l m}$ which has the highest energy (defined as the summation of all coefficients' square values). The corresponding frequency band of $\boldsymbol{V}_{l m}$ is defined as dominant band $F_{0}$ with a central frequency $f_{c}$. Suppose $x(t)=\sin \left(2 \pi f_{c} t\right)$, the regularity index $R_{i}$ can be written as

$$
R_{i}=\max _{\tau}\left|\frac{\int_{-\infty}^{\infty} x(t+\tau) y(t) d t}{\sqrt{\int_{-\infty}^{\infty} x^{2}(t) d t \times \int_{-\infty}^{\infty} y^{2}(t) d t}}\right|
$$

which is the maximum normalized cross-correlation between EEG signal $y(t)$ and $x(t)$. The higher value of $R_{i}$ indicates more similarity between $y(t)$ and $x(t)$, which also means that the power of EEG signal $\mathrm{y}(\mathrm{t})$ is concentrated in a narrow range near central frequency $f_{c}$. As such, the regularity index $R_{i}$ is a good representation of rhythmic synchronization. In the feature extraction step, $R_{i}$ is computed for each of the 18 channels and form a $18 \times 1$ dimensional feature vector for one EEG epoch.

The regularity index for the same EEG session as in Fig. 4 is plot in Fig. 5. It is demonstrated that the EEG frequency regularity is much higher in ictal state than in interictal state, which is consistent with the iii) of hypothesis we proposed in Section I. 


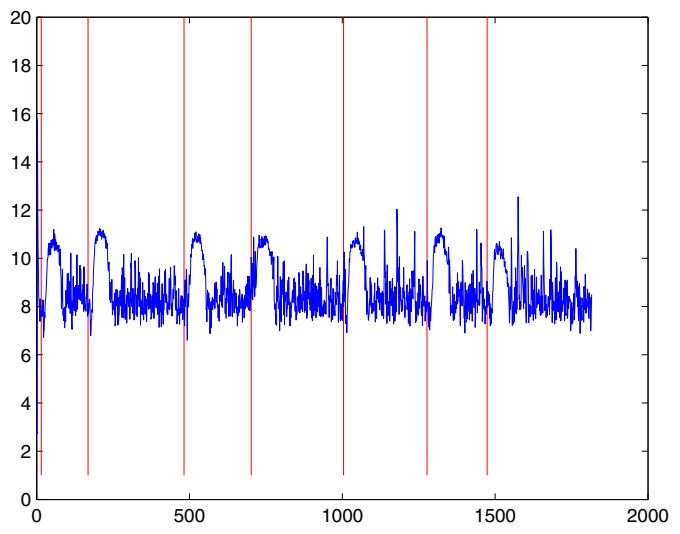

Fig. 5. Regularity index of an entire session. Red markings indicate seizures manually marked by EEG technician.

\section{E. Feature Selection and Classification}

We combine the spatiotemporal correlation, regularity index, power and entropy together as extracted feature vector, which has dimension of $278 \times 1$. This high-dimensional feature vector are reduced to 20-dimensional feature vector through feature selection using mutual information (MI) method based on best individual features with parzen window.

Support vector machine have recently demonstrated impressive performance in seizure prediction [4], [8]. In the classification stage of the seizure detector, the reduced feature vector is assigned to the ictal or interictal class by SVM classifier.

The seizure onset is declared when 3 consecutive $2 \mathrm{~s}$ epoches are classified as seizure. Requiring seizure activity to last for $6 \mathrm{~s}$ prior to declaring a seizure event helps avoid false alarm due to short-time seizure-like spikes in iterictal state.

\section{EXPERIMENTAL RESULTS}

\section{A. Performance Evaluation}

The performance of the seizure detector can be evaluated by specificity, sensitivity, accuracy and detection latency, as defined below:

$$
\begin{aligned}
\text { Sensitivity } & =\mathrm{TP} /(\mathrm{TP}+\mathrm{FN}) \\
\text { Specificity } & =\mathrm{TN} /(\mathrm{TN}+\mathrm{FP}) \\
\text { Accuracy } & =(\mathrm{TN}+\mathrm{TP}) /(\mathrm{TN}+\mathrm{TP}+\mathrm{FN}+\mathrm{FP})
\end{aligned}
$$

Where $\mathrm{TP}=$ true positive, $\mathrm{TN}=$ true negative, $\mathrm{FP}=$ false positiv and $\mathrm{FN}=$ false negative.

Consider $t_{i}$ is the time label of the end point of the first 3 consecutive ictal epochs in one seizure session marked by our method,$t_{l}$ is the seizure event start point marked by technician, the metric latency is defined as $t_{i}+2-t_{l}$.

\section{B. Leave-one-session-out Cross-validation}

For each patients, the entire session are firstly divided into subsessions based on number of seizure event. Each subsession start from the starting point of seizure event and end at starting point of next seizure event. The performance is evaluated through a 'leave one-session out' cross-validation, i.e., each time keep one subsession as test dataset, the rest subsessions as training dataset. For unbiased performance evaluation, the label-dependant feature selection and classification algorithms are trained only by training dataset. Then the test dataset is passed into the trained model to get results for performance evaluation.

\section{Results}

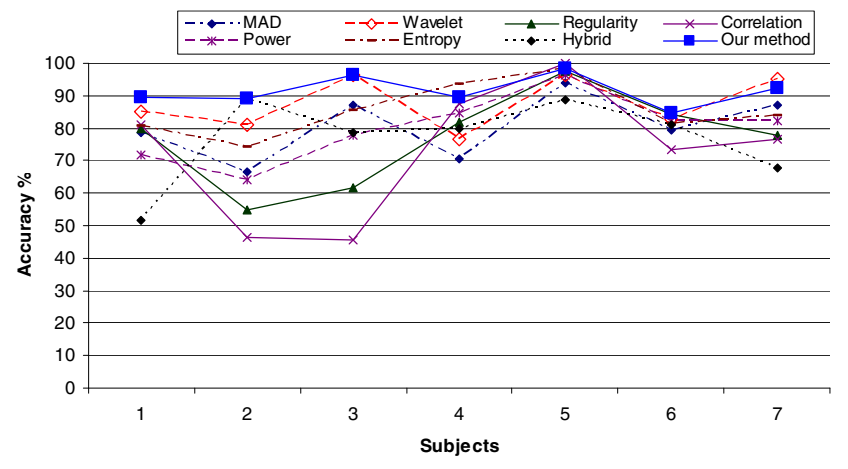

Fig. 6. Accuracy comparison for each subject

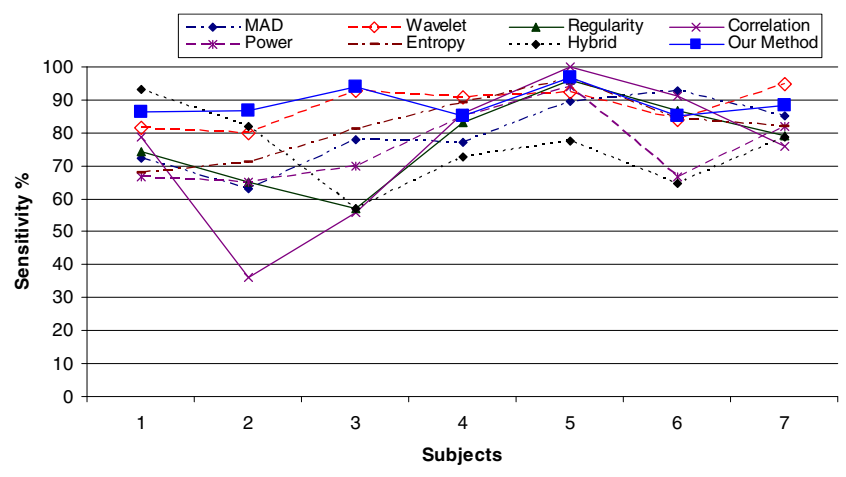

Fig. 7. Sensitivity comparison for each subject

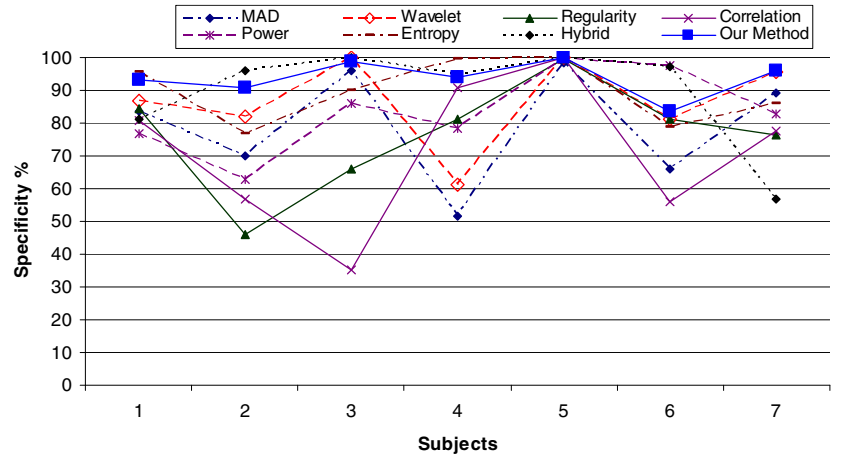

Fig. 8. Specificity comparison for each subject 


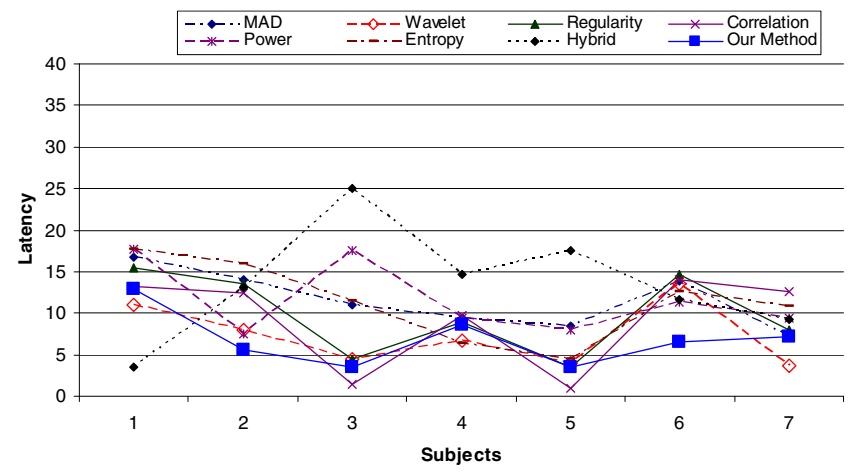

Fig. 9. Latency comparison for each subject

Figs. 6, 7, 8, 9 shows the results of the evaluation metrics, accuracy, sensitivity, specificity and latency, based on 8 different feature extraction methods: median absolute deviation (MAD) [3], wavelet coefficients (wavelet) [4], frequency regularity (regularity) [12], spatiotemporal correlation (correlation) [8], power (power) [1], entropy (entropy) [2], hybrid feature (hybrid) [6] and our own method. Results for each of the 7 patients are illustrated in these figures, from which we can see our proposed method shows an overall superiority over the rest. Although our method may not necessarily be the best method regarding each single subject, consider that some other method may give very high performance on one subject but give unsatisfactory performance on the other subjects, they are not quite suitable for real clinical application, for which system robustness is critical.

The average results of the 7 patients for the evaluation metrics: accuracy, sensitivity, specificity and latency, are shown in Table II. The best results for each metric are marked as bold typeface in the table.

For clearer illustration, the average results are plotted in Fig. 10. We can see that our method outperforms the rest methods refer to the average accuracy, sensitivity, specificity and latency. Furthermore, the standard deviation (illustrated as the error bar in the figure) of the results of our method are smaller than other methods which indicates higher robustness.

\section{CONCLUSION}

We proposed a hypothesis by investigating the fundamental mechanism of epileptic seizure. A robust seizure detection algorithm was developed based on this hypothesis. Spatiotemporal correlation structure that representing variance across EEG channels and time interval was combined with frequency regularity that representing frequency synchronization, to form a more robust seizure index. The combined index can capture

TABLE II

AVERAGE RESUlTS FOR COMPARISON

\begin{tabular}{|l|l|l|l|l|l|l|l|l|}
\hline Subjects & MAD(\%) & Wavelet(\%) & Regularity(\%) & Correlation(\%) & Power(\%) & Entropy(\%) & Hybrid(\%) & Our Method(\%) \\
\hline Accuracy & $80.48 \pm 9.74$ & $87.61 \pm 8.22$ & $76.84 \pm 14.36$ & $72.95 \pm 20.31$ & $79.90 \pm 10.19$ & $85.38 \pm 8.17$ & $76.66 \pm 13.11$ & $\mathbf{9 1 . 4 4} \pm 4.76$ \\
\hline Sensitivity & $79.68 \pm 10.31$ & $88.04 \pm 6.03$ & $77.31 \pm 13.21$ & $74.82 \pm 22.00$ & $75.68 \pm 11.34$ & $81.68 \pm 9.96$ & $75.12 \pm 11.85$ & $\mathbf{8 8 . 9 9} \pm 4.62$ \\
\hline Specificity & $79.25 \pm 17.29$ & $86.65 \pm 13.73$ & $76.40 \pm 16.72$ & $71.07 \pm 22.67$ & $83.45 \pm 12.60$ & $89.49 \pm 9.43$ & $77.95 \pm 37.16$ & $\mathbf{9 3 . 8 2} \pm 5.53$ \\
\hline Latency & $11.52 \pm 3.46$ & $7.33 \pm 3.79$ & $9.79 \pm 4.84$ & $9.22 \pm 5.60$ & $11.54 \pm 4.32$ & $11.34 \pm 4.76$ & $13.20 \pm 7.27$ & $\mathbf{6 . 8 2} \pm 3.26$ \\
\hline
\end{tabular}

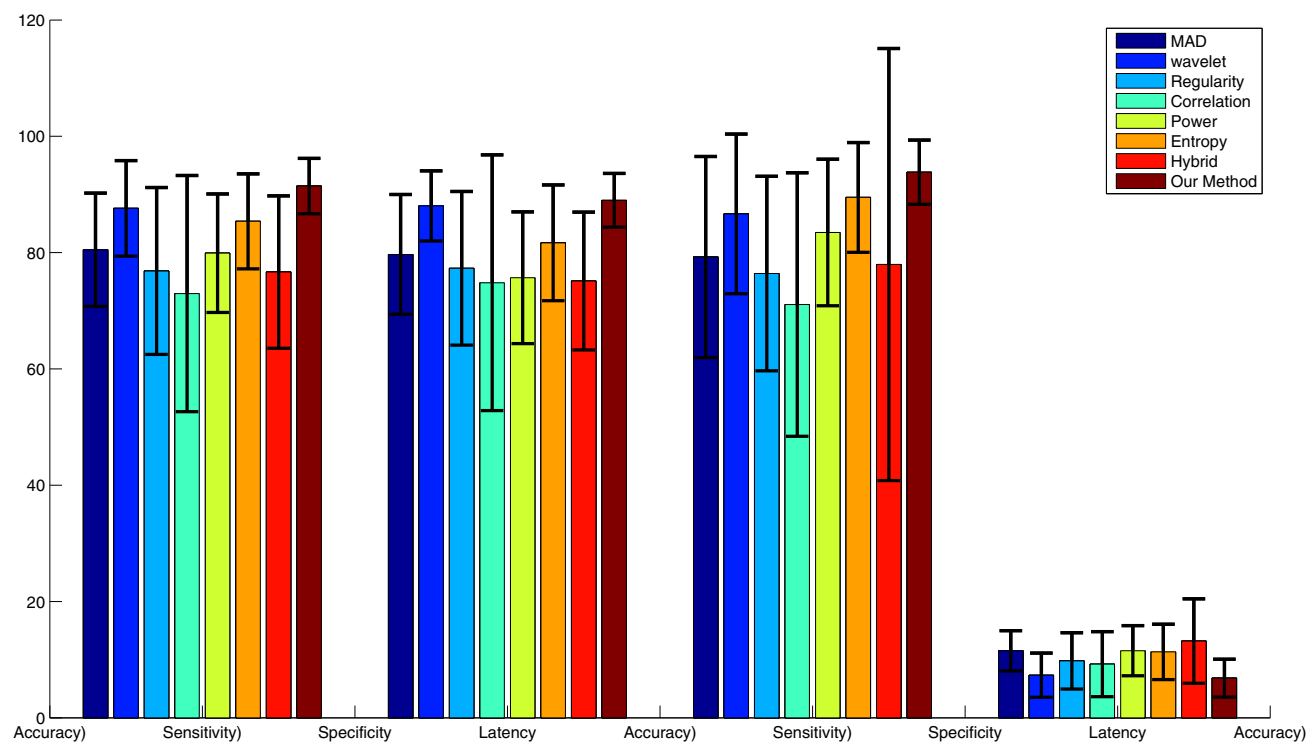

Fig. 10. Compare performance metrics among algorithms 
both amplitude and frequency changes. In addition, since the combined feature vector is high-dimensional with redundancy, feature selection was performed using mutual information (MI) based method to obtain an optimized set of features for more compact feature vector, which was later classified by SVM. In experiments based on 43 seizure events from 7 epilepsy patients, $91.44 \%$ average accuracy and $6.82 \mathrm{~s}$ latency was obtained using the proposed method. It was demonstrated that the proposed method outperformed other commonly used methods in both accuracy aspect and robustness aspect. This may due to the fact that our feature extraction method captured spatiotemporal features and spectral features at the same time so that the seizure indexes used are more sensitive and robust. The hypothesis we proposed was well verified through our data analysis as well. We will continue to analyze much larger dataset of epilepsy patients in order to develop more robust subject-independent seizure detection system in the future.

\section{REFERENCES}

[1] B. Litt, R. Esteller, and J. Echauz, "Epileptic seizures may begin hours in advance of clinical onset: a report of five patients," Neuron, vol. 30, pp. 51-64, 2001.

[2] S. P. Kumar, N. Sriraam, P. G. Benakop, and B. C. Jinaga, "Entropies based detection of epileptic seizures with artificial neural network classifiers," Expert Systems with Applications, vol. 37, no. 4, pp. 3284 3291, 2010.

[3] M. Bedeeuzzaman, O. Farooq, and Y. U. Khan, "Dispersion measures and entropy for seizure detection," in 2011 IEEE International Conference on Acoustics, Speech and Signal Processing (ICASSP), 2011, pp. 673-676.

[4] A. Shoeb, H. Edwards, J. Connolly, B. Bourgeois, S. T. Treves, and J. Guttag, "Patient-specific seizure onset detection," Epilepsy \& Behavior, vol. 5, no. 4, pp. 483-498, 2004.

[5] Y. U. Khan and O. Farooq, "Autoregressive features based classification for seizure detection using neural network in scalp electroencephalogram," International Journal of Biomedical Engneering and Technology, vol. 2, no. 4, pp. 370-381, 2009.

[6] A. B. Gardner, A. M. Krieger, G. Vachtsevanos, and B. Litt, "One-class novelty detection for seizure analysis from intracranial EEG," Journal of Machine Learning Research, vol. 7, pp. 1025-1044, 2006.

[7] Y. Pan, S. S. Ge, F. R. Tang, and A. A. Mamun, "Detection of epileptic spike-wave discharges using SVM," in Proceedings of the 2007 IEEE International Conference on Control Applications, Suntec City, Singapore, 1-3 October 2007, pp. 467-472.

[8] J. R. Williamson, W. B. Daniel, and W. B. David, "Epileptic seizure prediction using the spatiotemporal correlation struction of intracranial EEG," in 2011 IEEE International Conference on Acoustics, Speech and Signal Processing (ICASSP), 2011, pp. 665-668.

[9] J. Gotman, "Automatic recognition of epileptic seizures in the EEG," Electroencephalography and Clinical Neurophysiology, vol. 54, pp. 530$540,1982$.

[10] M. E. Saab and J. Gotman, "A system to detect the onset of epileptic seizures in scalp EEG," Clinical Neurophysiology, vol. 116, pp. 427442, 2005.

[11] K. Schindler, H. Leung, C. E. Elger, and K. Lehnertz, "Assessing seizure dynamics by analysing the correlation structure of multichannel intracranial EEG," Brain, vol. 130, pp. 65-77, 2006.

[12] A. S. Zandi, G. A. Dumont, M. Javidan, R. Tafreshi, B. A. Macleod, C. R. Ries, and E. Puil, "A novel wavelet-base index to detect epileptic seizures using scalp EEG signals," in 30 th Annual International IEEE EMBS Conference, 2008, pp. 919-922.

[13] A. S. Zandi, M. Javidan, G. A. Dumont, and R. Tafreshi, "Automated real-time epileptic seizure detection in scalp EEG recordings using an alsorithm based on wavelet packet transform," IEEE Transactions on Biomedical Engineering, vol. 57, no. 7, pp. 1639-1651, 2010. 